\title{
Effect of Antenna Directivity on the Accuracy of Fingerprint-based Localisation
}

\author{
Qiao Cheng \\ q.cheng@qmul.ac.uk \\ Akram Alomainy \\ a.alomainy@qmul.ac.uk \\ Yang Hao
y.hao@qmul.ac.uk \\ School of Electronic Engineering and Computer Science \\ Queen Mary University of London \\ London, United Kingdom
}

\begin{abstract}
This paper investigates the effect of antenna directivity on the accuracy of fingerprint-based indoor localisation systems. The proposed method adopts received signal strength which was derived by ray tracing techniques applied to 3D indoor model for location determination. Antennas with different radiation patterns and different orientations are implemented for comparative study. Numerical results show that, by utilizing the geometry of the indoor environment, directional antennas can help increase the uniqueness of the fingerprints and hence improve the localisation accuracy.
\end{abstract}

\section{Categories and Subject Descriptors}

C.2.1 [Computer Communication Networks]: Network Architecture and Design-Wireless communication

\section{General Terms}

Algorithms, Design

\section{Keywords}

Fingerprinting, indoor localisation, antenna directivity, antenna orientation

\section{INTRODUCTION}

Location information plays an important part for many applications like navigation, surveillance, entertainment, health, etc. However, localisation accuracy in indoor environment like offices, schools and hospitals is faced with non-line-ofsight transmission, severe multipath fading and shadowing effects due to obstacles like walls, furniture and human bodies. The performance of the traditional outdoor triangulation approaches is greatly impaired in indoor environment. Alternatively, most indoor localisation systems adopted twophase radio frequency ( $\mathrm{RF}$ ) fingerprinting for location determination [2]. RF fingerprinting localisation (FL) is a low cost and easy to implement technique that leverages the prevalent Wi-Fi infrastructure.
During the last decade, researchers mainly strived to either exploit sophistic algorithms for better accuracy in the online phase $[5,6,3]$ or develop training techniques that attempt to shorten the calibration time in the offline phase $[11,10]$. All those efforts are trying to improve the FL system in a numerical/algorithmic way without changing the available Wi-Fi infrastructure. However, few people have considered utilizing the physical parameters of the system. Antenna, for instance, which plays a vital role in wireless communication, can have huge impact on the received signal strength (RSS) in FL. In [4], the author analysed the influence of the antenna orientation on the calibration of indoor propagation model, which shows the accuracy may be greatly jeopardized if the antenna orientations are not considered. A directional antenna based technique was proposed in [8] for wireless sensor networks. However, this is a hybrid method using angle of arrival information which renders little insight into FL.

In this paper, we analyse the effects of directional antennas on FL. In particular, we investigate a dipole antenna and three directional antennas with different beam width. Eight orientations of the antenna were taken into consideration. Ray tracing based electromagnetic (EM) propagation software [1] was adopted to simulate indoor RF propagation instead of empirical propagation model.

\section{THEORETICAL BASIS}

The main procedure of FL is to collect the RSS as fingerprints at predefined locations which are called reference points (RPs) and then build a fingerprint database. The location of the target is then determined by matching runtime fingerprint to the database using certain algorithms. As its name implies, FL relies on the uniqueness of each fingerprint, which means ambiguities among the fingerprints should be highly avoided [9]. In other words, if many locations have similar fingerprints, the localisation algorithm will not be able to distinguish among those locations. Therefore, the ultimate goal of FL is to maximize the fingerprint difference among the RPs.

The RSS at the antenna is determined by the superposition of EM waves with different magnitudes and phases from all possible directions. In a specified environment, antenna's RSS along one path can vary in different scales across different directions. Fig. 1 gives an example of the RSS in a long corridor. The first three colour bars represent the fingerprints from omnidirectional antenna, directional antenna facing east and directional antenna facing north, re- 
(a)

(b)

(c)

(d)

Figure 1: RSS of (a) omnidirectional antenna, (b) directional antenna facing east and (c) directional antenna facing north. (d) RSS intensity.

spectively. The fourth bar indicates the RSS varies from $-85.8 \mathrm{dBm}$ to $-18.8 \mathrm{dBm}$. It is obvious that directional antenna has fewer similarities among the fingerprints than omnidirectional antenna.

Therefore, we propose to use directional antennas such that the direction of the maximum gain is parallel to the direction where RSS has rapid variation. Furthermore, directional antennas usually have higher gain than omnidirectional antennas and hence can capture more signal energy in the desired direction. This property guarantees a more stable signal reception against the complex indoor environment which is quite desirable for the FL. In this way, the number of similar fingerprints is minimized and hence the probability of wrong estimation is reduced.

\section{NUMERICAL ANALYSIS SET-UP}

The effectiveness of proposed method employing directional antennas is studied via ray racing technique followed by MATLAB post-processing. We built a $17 m \times 46 m$ 3D office environment with a long corridor as shown in Fig. 2. Concrete walls, ceilings and wooden doors are assumed in the simulation. In this case, the receiver antenna's RSS should be dominated by the EM waves along the corridor and varies less rapidly than only receiving EM waves that are perpendicular to the corridor. Within the simulation setup, four half wave dipole transmitters with $20 \mathrm{dBm}$ transmit power are placed in separate rooms. For the receiver, we adopt a dipole antenna and three ideal directional antennas with different beam width. Fig. 3 shows the $3 \mathrm{D}$ radiation pattern of the four receiver antennas. For each directional

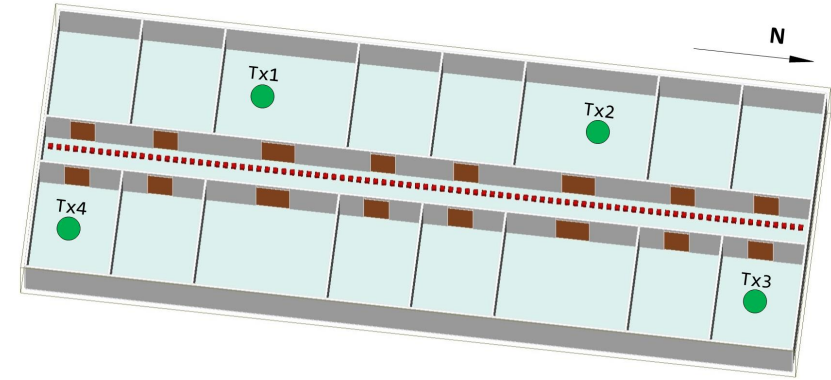

Figure 2: $17 m \times 46 m$ test bed with receivers (red) and transmitters (green).

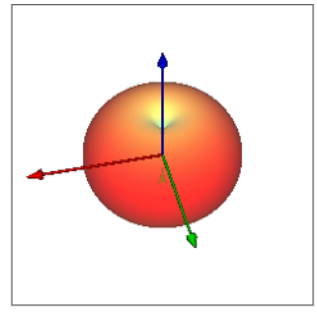

(a)

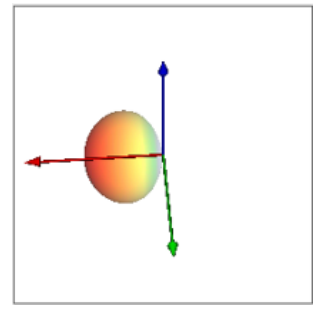

(c)

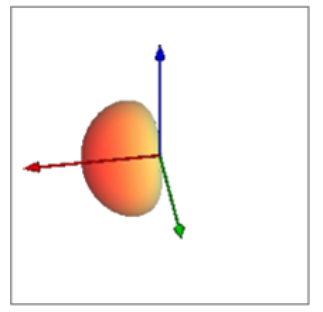

(b)

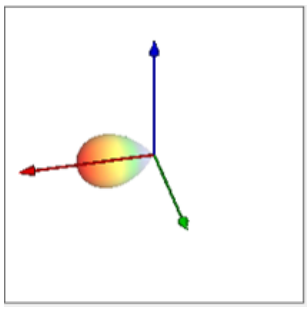

(d)
Figure 3: 3D radiation patterns of (a) dipole, (b) $90^{\circ}$ beam antenna, (c) $60^{\circ}$ beam antenna and (d) $30^{\circ}$ beam antenna.

antenna, eight orientations facing east, west, north, south, northwest, southwest, northeast and southeast are tested. All the antennas are placed $2 m$ over the ground with vertical polarization and the operating frequency is set to 2.4 $\mathrm{GHz}$ to simulate the indoor Wi-Fi environment. During the training phase, the radio maps are generated from $91 \mathrm{RPs}$ placed in the middle of the corridor with a spacing of $0.5 \mathrm{~m}$. During the runtime phase, a grid of 1140 receivers are placed near the RPs with a spacing of $20 \mathrm{~cm}$ to obtain runtime test points.

\section{SIMULATION RESULTS}

In order to fairly compare the precision of different schemes, the nearest neighbour (NN) algorithm [2] is applied. NN algorithm first computes the Euclidean distance between the runtime fingerprint and fingerprint database, and then the location of the fingerprint that corresponds to the minimum Euclidean distance is chosen as the estimated location. For computation simplicity, we don't consider receiver sensitivity and assume all the signals can be received. For ease of comparison, the directional antennas facing the corridor walls (east and west) are referred to as scheme 1, directional antennas parallel to the corridor (south and north) are referred to as scheme 2 and the rest as scheme 3.

In Fig. 4, it is noticed that scheme 1 and scheme 2 achieve the best and the worst accuracy respectively. The performance of Scheme 3 is between scheme 1 and scheme 2 since it combines the advantage of scheme 1 and disadvantage of scheme 2. All 3 schemes, except for $30^{\circ}$ beam facing north, achieve lower mean location error in comparison with dipole antenna case, which indicates the effectiveness of directional antenna. Further, for scheme 1, the more directional the antenna is, the better accuracy the system achieves. On the contrary, in scheme 2, higher directivity means less accuracy. This interesting result suggests that increased directivity does not always give better accuracy. The orientation 


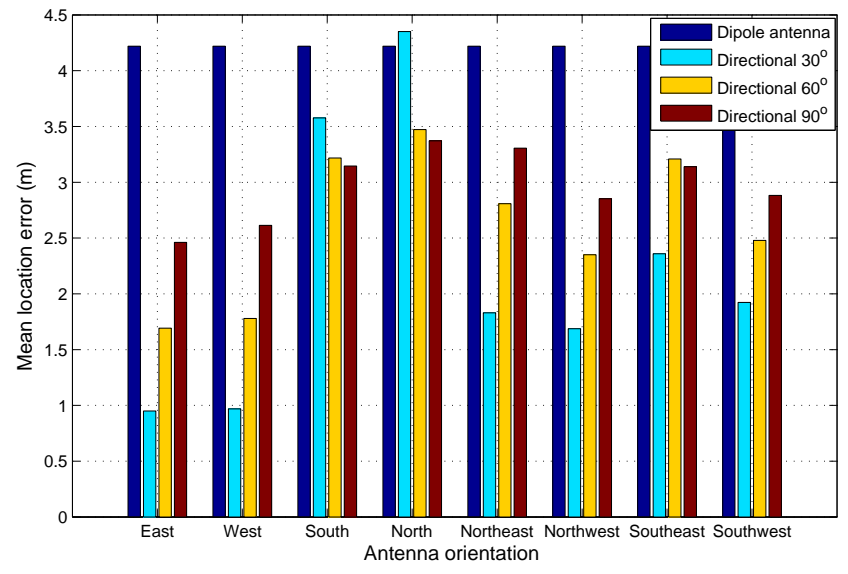

Figure 4: Mean location error versus different antennas.

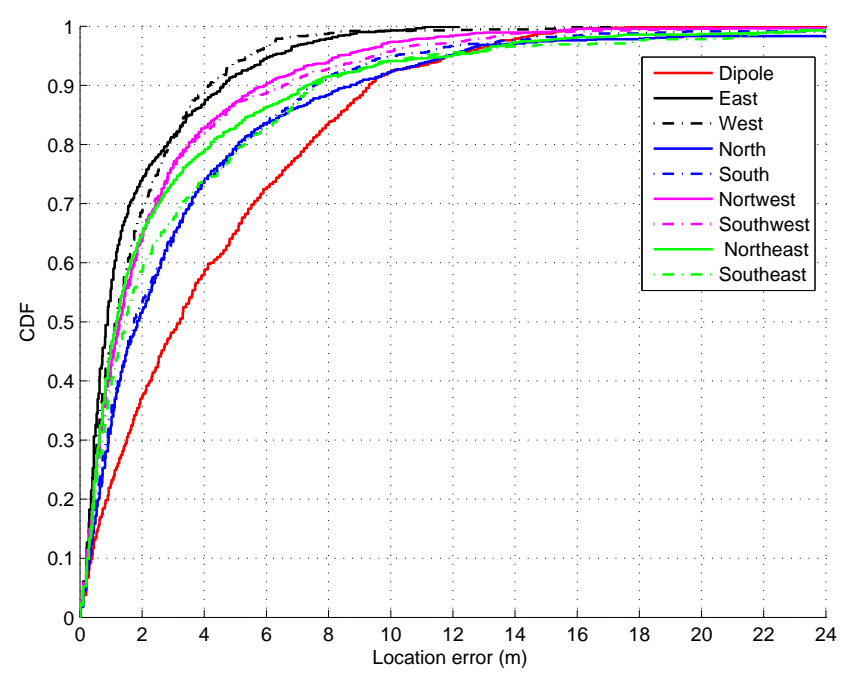

Figure 5: CDF comparison of dipole and $60^{\circ}$ beam directional antenna.

of the antenna should be chosen carefully such that directional antenna can achieve the best performance.

More specifically, Fig. 5 illustrates the localisation error distributions of the $60^{\circ}$ beam directional antenna across each direction. Note that the CDF curve only shows the location error between $0 m$ and $24 m$ for better visualization. Scheme 1 bounds the $90^{t h}$ percentile errors to only about $5 \mathrm{~m}$, while scheme 2 and dipole antenna case drop to 80 percentile and 65 percentile, respectively, to maintain the same location error. Scheme 3 is again between scheme 1 and scheme 2, which agrees with previous results. It can also be noticed that scheme 2 has higher probability of achieving huge location error, which explains why the $30^{\circ}$ beam directional antenna facing north, as shown in Fig. 4, has larger mean location error than the dipole antenna case.

In reality, a signal will typically experience random varia-

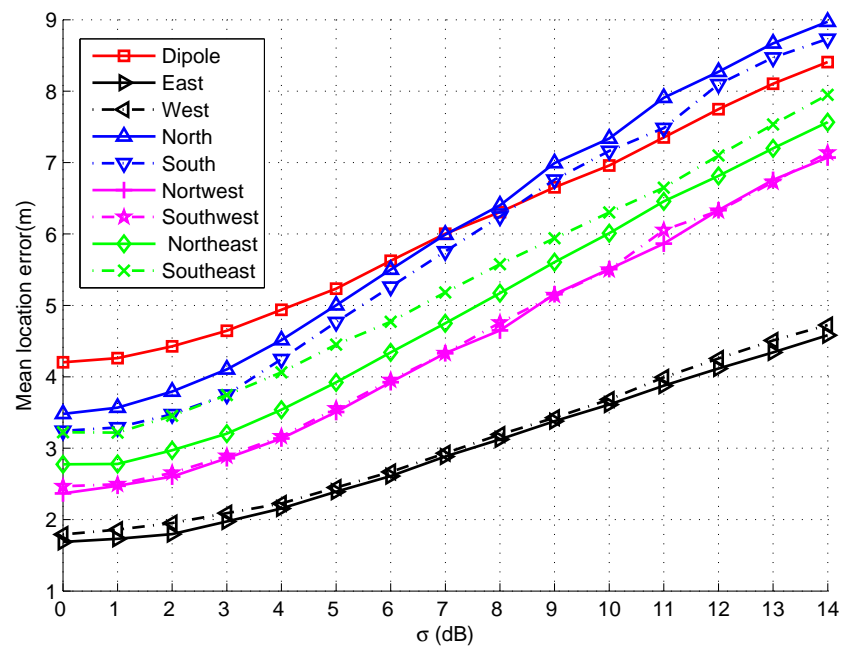

Figure 6: Mean location error vs. noise standard deviation $\sigma\left(60^{\circ}\right.$ beam directional antenna).

tion due to shadow fading [7]. This shadowing effect is examined by manually adding Gaussian noise with zero mean and variance $\sigma^{2}$ to the runtime phase test points, where $\sigma$ varies from $0 \mathrm{~dB}$ to $14 \mathrm{~dB}$. For each $\sigma$ value, 20000 points have been randomly and independently chosen from the runtime test points for simulation. Fig. 6 demonstrates the mean location error for dipole antenna and $60^{\circ}$ beam directional antenna with 8 orientations as a function of $\sigma$. We observe that as the noise increases, the localisation accuracy decreases. Further, scheme 1 and scheme 3 have better accuracy than dipole antenna case. However, scheme 2 becomes worse than dipole antenna case when the $\sigma$ is greater than $8 \mathrm{~dB}$.

Fig. 7 presents the effect of antenna directivity on the mean location error versus noise standard deviation $\sigma$. For simplicity, only 4 antenna orientations have been shown here. In $7(\mathrm{a}), 7(\mathrm{c})$ and $7(\mathrm{~d})$, higher directivity antennas are more robust to the noise. In 7(b), on the contrary, higher directivity antennas introduce more location errors. This behaviour is expected since by steering the antenna beam parallel to the corridor, the higher directivity the antenna is, the less variation the fingerprints have.

\section{CONCLUSIONS}

In this paper, we propose to use directional antennas to improve the accuracy of fingerprint-based localisation. The effect of antenna directivity and orientation has been demonstrated and analysed. Numerical analysis shows when the direction of the maximum gain of the antenna align with the direction where RSS has rapid variation, the high directional antennas can achieve better accuracy and are more robust to shadowing effect. Specifically, with a $60^{\circ}$ beam directional antenna, a $25 \%$ increase of probability of location error under $5 \mathrm{~m}$ can be achieved compared to omnidirectional antenna. However, if the condition does not satisfy, high directivity may deteriorate the performance. Therefore, to ensure location accuracy as well as signal coverage, a trade-off can be made by choosing a moderate directional 


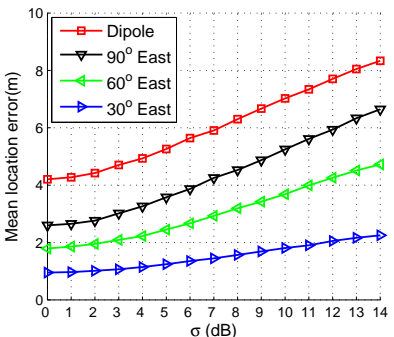

(a)

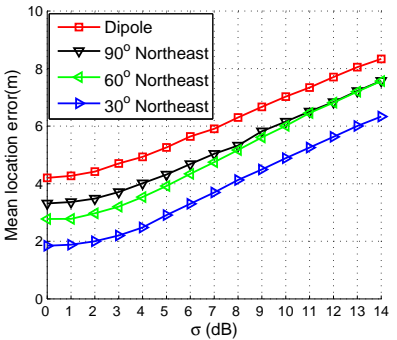

(c)

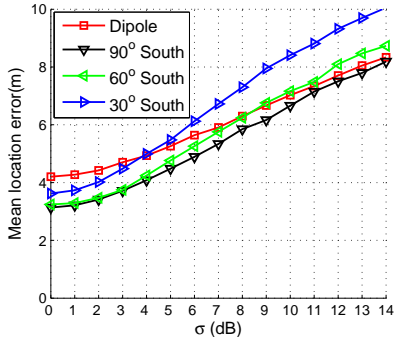

(b)

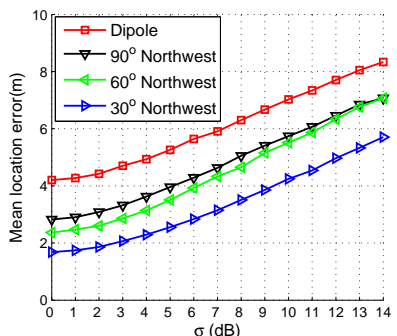

(d)
Figure 7: Mean location error vs. noise standard deviation $\sigma$ : (a) dipole and east, (b) dipole and south, (c) dipole and northeast and (d) dipole and northwest.

\section{antenna.}

As for the applications, this technique can be considered for better accuracy where omnidirectional antenna is not mandatory. For example, for a patients monitoring system in hospital, accurate tracking of the patients is the first priority. Therefore, directional antennas can be adopted for the patient's wireless tag. Even for current mobile devices where antennas are designed to be omnidirectional, the directivity may be changed slightly due to the human body presence. This property can then be utilized to by facing the mobile device to the appropriate direction. Moreover, in future $5 \mathrm{G}$ communication systems, phased array can be integrated into the mobile devices thanks to the higher frequency band. Electronically beam steering of the phased array makes accurate localisation even easier and simpler.

\section{REFERENCES}

[1] Wireless EM propagation software - wireless InSite remcom.

[2] P. Bahl and V. Padmanabhan. Radar: an in-building rf-based user location and tracking system. In INFOCOM 2000. Nineteenth Annual Joint Conference of the IEEE Computer and Communications Societies. Proceedings. IEEE, 2000.

[3] Q. Cheng, M. Munoz, A. Alomainy, and Y. Hao. Compressive sensing applied to fingerprint-based localisation. In RF and Wireless Technologies for Biomedical and Healthcare Applications (IMWS-Bio), 2014 IEEE MTT-S International Microwave Workshop Series on, Dec 2014.

[4] B. Dil and P. Havinga. Rss-based localization with different antenna orientations. In Telecommunication Networks and Applications Conference (ATNAC),
2010 Australasian, pages 13-18, Oct 2010.

[5] S.-H. Fang and T.-N. Lin. Principal component localization in indoor wlan environments. Mobile Computing, IEEE Transactions on, 11(1):100-110, Jan 2012.

[6] C. Feng, W. Au, S. Valaee, and Z. Tan. Received-signal-strength-based indoor positioning using compressive sensing. Mobile Computing, IEEE Transactions on, 11(12):1983-1993, Dec 2012.

[7] A. Goldsmith. Wireless Communications. Cambridge University Press, 2005.

[8] F.-Y. L. Jehn-Ruey Jiang, Chih-Ming Lin and S.-T. Huang. Alrd: Aoa localization with rssi differences of directional antennas for wireless sensor networks. International Journal of Distributed Sensor Networks, 2013.

[9] K. Kaemarungsi. Design of Indoor Positioning Systems based on Location Fingerprinting Technique. PhD thesis, University of Pittsburgh, Pittsburgh, Feburary 2005.

[10] S. Nikitaki, G. Tsagkatakis, and P. Tsakalides. Efficient training for fingerprint based positioning using matrix completion. In Signal Processing Conference (EUSIPCO), 2012 Proceedings of the 20th European, pages 195-199, Aug 2012.

[11] A. Rai, K. K. Chintalapudi, V. N. Padmanabhan, and R. Sen. Zee: Zero-effort crowdsourcing for indoor localization. In Proceedings of the 18th Annual International Conference on Mobile Computing and Networking, Mobicom '12, 2012. 